\title{
A General View of the Role of Specific nutrients in the Prevention of Cardiovascular Diseases
}

\author{
Maralla Swaroopa $^{* 1}$ and D. Bharathi ${ }^{2}$ \\ *1UGC Post Doc Fellow in Biomedicine, Division of Zoology, Department of Sericulture, Sri Padmavathi Mahila University, Tirupati-517502, \\ Andhra Pradesh, India. \\ ${ }^{2}$ Professor, Department of Sericulture, Sri Padmavathi Mahila University, Tirupati-517502, Andhra Pradesh, India.
}

\begin{abstract}
Nearly, 15.3 million people are estimated to die from cardiovascular diseases every year; and this represents one-third of all global deaths from all causes. Lifestyle factors, including nutrition, play an important role in the etiology of Cardiovascular Disease (CVD). A high intake of dietary fats strongly influences the risk of developing cardiovascular disease (CVD). Diet offers incredible opportunities for prevention of cardiovascular disease. Dietary patterns are useful for reducing CVD risk factors, and some have also shown a favourable effect on plaque regression and CVD mortality. Thus, every patient need to adopt a dietary approach that conforms to his or her personal preferences; however, implementing relevant and successful dietary changes is the greatest challenge for preventive cardiovascular medicine.
\end{abstract}

Keywords: Dietary Patterns, Cardiovascular Disease, Risk Factors, Mortality and Preventive Cardiovascular Medicine.

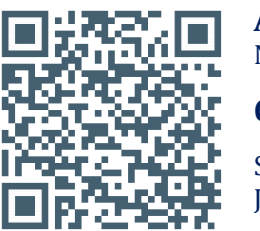

Article Info: Received 30 Sep, 2018; Review Completed 29 Oct 2018; Accepted 30 Oct 2018; Available online 15 Nov 2018

Cite this article as:

Swaroopa M, Bharathi D, A General View of the Role of Specific nutrients in the Prevention of Cardiovascular Diseases, Journal of Drug Delivery and Therapeutics. 2018; 8(6):310-313 DOI: http://dx.doi.org/10.22270/jddt.v8i6.2026

*Address for Correspondence:

Swaroopa. Maralla, UGC Post Doc Fellow in Biomedicine, Division of Zoology, Department of Sericulture, Sri Padmavathi Mahila University, Tirupati-517502, Andhra Pradesh, India.

\section{INTRODUCTION}

Cardiovascular disease (CVD) is a class of diseases that involve the heart or blood vessels ${ }^{1}$ (Shanthi et al., 2011). Cardiovascular disease includes Coronary Artery Diseases (CADs) such as angina and myocardial infarction (commonly known as a heart attack).

Amongst others include:

- Atherosclerosis, which occurs when fatty deposits clog and harden arteries,

- Coronary Heart Disease (CHD), caused by the reduced blood supply to the heart muscle,

- Stroke, caused by inadequate blood flow to the brain leading to the death of brain cells,

- Hypertension, occurs when blood pressure is higher than the normal range,

- Cardiac Arrhythmias, which are irregular or abnormal heartbeats.

\section{Risk factors for CVD}

The cause of most CVD is the gradual narrowing or blockage of blood vessels. This process is called atherosclerosis and is due to the build-up of fatty deposits in the lining of blood vessels.

There are a number of factors that are associated with developing CVD. Risk factors include:

- High blood cholesterol;

- High blood pressure;

- Smoking;

- Physical inactivity;

- Being overweight;

- Excessive alcohol consumption;

- Diabetes; and

- Depression.

Many of these risk factors can be prevented and managed by lifestyle changes. A family history of CVD, older age and being male also increases the risk of developing CVD. 


\section{Diet and Cardiovascular disease}

The role of diet is crucial in the development and prevention of cardiovascular disease. Diet is one of the key things that will impact all other cardiovascular risk factors. Diet can affect the vast majority of modifiable risk factors for cardiovascular disease, which are now identified as explaining a very large part of the variability in the occurrence of a first acute myocardial infarction. Modulation of vascular inflammation is likely the most relevant common pathogenetic step by which nutritional factors influence cardiovascular disease. Vascular inflammation is influenced by excess caloric intake (obesity, insulin resistance), alcohol, several vitamins, dietary antioxidants, and n-3 PUFAs.

Comparisons between a diet low in saturated fats, with plenty of fresh fruit and vegetables, and the typical diet of someone living in the developed world show that in the former there is a $73 \%$ reduction in the risk of new major cardiac events.

\section{Fat}

Research makes it clear that abnormal blood lipid (fat) levels have a strong correlation with the risk of coronary artery disease, heart attack and coronary death. A diet high in saturated fats (e.g. cheese) and trans fats (often used in cakes, cookies and fast food) leads to high levels of cholesterol.

Saturated fats are found in animal products. Trans fats are oils that have been hydrogenated to turn them into semihard fats. Hydrogenated fat is found in processed food like shop-bought cakes, biscuits, stock cubes and a range of other products. Saturated and trans fats raise cholesterol levels in the blood, which in turn can lead to atherosclerosis.

Unsaturated fats, polyunsaturated and monounsaturated are beneficial for heart health. They are present in fish, nuts, seeds and vegetables. The essential fatty acids omega3 and omega- 6 are found in oily fish and in nuts and seeds.

But it is important to note that Saturated fat intake should not exceed $10 \%$ of total energy and for high-risk groups, like people with diabetes, total fat intake should be $7 \%$ or less of total energy.

\section{Sodium}

High blood pressure (hypertension) is a major risk factor for cardiovascular disease. If you have a diet high in sodium you risk hypertension.

It has been estimated that a universal reduction in dietary intake of sodium by about $1 \mathrm{~g}$ of sodium a day, about $3 \mathrm{~g}$ of salt, would lead to a $50 \%$ reduction in the number of people needing treatment for hypertension. The same decrease would lead to a $22 \%$ drop in the number of deaths resulting from strokes and a $16 \%$ fall in the number of deaths from coronary heart disease.

\section{Fruits and vegetables}

Eating a diet high in fresh fruits and vegetables protects heart health. Low fruit and vegetable intake accounts for about $20 \%$ of cardiovascular disease worldwide. Fruit and vegetables contain components that protect against heart disease and stroke.

\section{Wholegrain cereals}

Whole grains contain folic acid, B vitamins and fiber, all of which are important protectors against heart disease.

\section{Fish}

In countries where fish consumption is high there is a reduced risk of death from cardiovascular mortality.

\section{Nuts}

Eating nuts regularly is associated with decreased risk of coronary heart disease.

\section{Soy}

There is evidence that soy has a beneficial effect on blood lipid levels. Eating $47 \mathrm{~g}$ of soy protein a day led to a $9 \%$ drop in total cholesterol and a $13 \%$ reduction in LDL cholesterol in one study of people without any heart disease.

\section{Alcohol}

Only moderate consumption of Alcohol during occasional drinking may protect heart, but this version is not supported by any investigational results.

\section{Try a DASH of healthy eating}

Research has demonstrated that blood pressure and cholesterol can be lowered by following an eating plan called the Dietary Approaches to Stop Hypertension (DASH).

In fact, in people with slightly raised blood pressure, the DASH plan lowered blood pressure by an amount similar to that expected from medication. The DASH eating plan is low in fat and salt, and contains plenty of fruits, vegetables and three serves of mainly low fat dairy foods. Blood pressure can begin to fall after just two weeks on this eating plan. The DASH eating plan was also found to lower total cholesterol by $7 \%$ and LDL cholesterol by $9 \%$.

\section{Dietary Effects on Cardiovascular Risk Factors \& Outcomes}

- Dietary habits affect multiple CV risk factors, including both established risk factors (systolic and diastolic blood pressure, LDL cholesterol levels, HDL cholesterol levels, glucose levels, and obesity/weight gain) and novel risk factors (e.g., inflammation, cardiac arrhythmias, endothelial cell function, triglyceride levels, lipoprotein[a] levels, and heart rate).

- A DASH dietary pattern with low sodium reduced systolic blood pressure by $7.1 \mathrm{~mm} \mathrm{Hg}$ in adults without hyper-tension, and by $11.5 \mathrm{~mm} \mathrm{Hg}$ in adults with hypertension.

- $\quad$ For each $2 \%$ of calories from trans fat was associated with a $23 \%$ higher risk of coronary heart disease.

- Each daily serving of fruits or vegetables was associated with a $4 \%$ lower risk of CHD and a $5 \%$ lower risk of stroke.

- Greater whole grain intake (2.5 compared with 0.2 servings per day) was associated with a $21 \%$ lower risk of CVD events, with similar estimates for specific CVD outcomes such as heart disease, stroke and fatal CVD.

- Fish consumption was associated with significantly lower risk of CHD mortality. In contrast, each $50 \mathrm{~g}$ serving per day of processed meats was associated with higher incidence of both coronary heart disease and diabetes mellitus.

\section{Control of Major CHD Risk Factors}

Coronary heart disease risk is significantly influenced by the development of comorbid diet-related conditions, including abdominal obesity, high blood pressure, and 
elevated blood glucose concentrations. A combination of these conditions (along with elevated triglycerides and/or low HDL) is known as metabolic syndrome. The presence of metabolic syndrome predicts CHD more strongly than its individual components do ${ }^{2}$ (McNeill et al., 2005).

Preventing excess weight gain can lower the risk for coronary artery disease. Accumulation of abdominal fat in particular is associated with the severity of coronary atherosclerosis $^{3}$ (Kortelainen and Sarkioja, 1997). Studies have shown that obesity is a predictor of acute coronary events 4 (Suwaidi et al., 2001). Loss of excess body weight also reduces the inflammatory marker $\mathrm{C}$-reactive protein and the proinflammatory cytokine interleukin 6 , both of which have been correlated with CHD risk ${ }^{5,} 6$ (O'Brien et al., 2005; Kopp et al.,2003).

Blood pressure: Reductions in blood pressure to optimal levels prevent an estimated 37\% of CHD events in men and $56 \%$ in women 7 (Wong et al., 2003).

Blood glucose concentrations: Chronically elevated blood glucose increases risk for coronary disease ${ }^{8}$ (Liu and Willett, 2002). Even in persons without diabetes, impaired glucose tolerance is predictive of greater risk for cardiovascular morbidity ${ }^{9}$ (Qiao et al., 2003), and normalization of postprandial hyperglycemia reduces cardiovascular events in these patients ${ }^{10}$ (Gerich, 2005).

Exercise: Regular exercise reduces cardiovascular mortality in patients with established coronary heart disease $^{11}$ (Thompson and Lim, 2003), particularly if the activity is sufficiently intense ${ }^{12}$ (Tanasescu, 2002). Current recommendations suggest a minimum of 30 minutes of moderately vigorous physical activity every day. Before a CHD patient starts an exercise regimen, however, a physical examination is essential, with particular attention to cardiac function, joints, and feet.

\section{Role of Nutrition in the control of four of the risk factors for cardiovascular disease:}

1. High blood fats (cholesterol, triglycerides)

2. High blood pressure

3. Overweight and obesity; and

4. Diabetes

In many cases, these risk factors can be reduced or avoided with lifestyle changes.

\section{High blood fats (cholesterol, triglycerides)}

A high blood cholesterol level raises the risk of atherosclerosis. Atherosclerosis is a process which causes a narrowing of the inside of arteries and a reduced blood flow. This can begin at an early age. The Heart Foundation recommends a blood cholesterol level less than $5.5 \mathrm{mmol} /$ litre as being desirable for adults. High blood cholesterol has been directly linked to an eating pattern high in fat, particularly saturated fat.

Fats in food are a mixture of three main types. They are:

- $\quad$ Saturated

- Mono-unsaturated; and

- Polyunsaturated.

Saturated fats are generally found in animal fats, like meat fat, poultry skin, full-cream dairy products and many processed foods tend to raise blood cholesterol and should be limited where possible. Vegetable oils, coconut and palm oil are also high in saturated fat.

Mono-unsaturated fats found in some oils like canola and olive oil, mono-unsaturated margarine, avocados, nuts and seeds may have a small lowering effect on blood cholesterol levels if they are used to replace saturated fats.

Polyunsaturated fats in oils such as sunflower, safflower and soybean, and polyunsaturated margarines, nuts and seeds and fish oils can also help lower blood cholesterol.

In summary it is important to limit all fats especially saturated fats as much as possible to lower blood cholesterol levels. This can be done by:

- Reducing total fat, especially saturated fats (fatty meats, full cream dairy products, butter, two vegetable oils - coconut and palm oil, and most fried take-aways and commercially baked products.

- Eating more bread, cereals, rice, pasta, vegetables, fruits, and legumes (dried peas, beans and lentils).

\section{High blood pressure}

When the pressure of the blood in the arteries is too high it puts a strain on the heart because it has to pump harder. The causes of high blood pressure are not well understood but being overweight, drinking too much alcohol, not being physically active and eating too much salt or salty foods are linked to this condition.

To help lower blood pressure, it is important to:

- enjoy healthy eating

- be physically active

- $\quad$ lose excess body fat

- limit alcohol intake; and

- $\quad$ Reduce salt intake.

\section{Overweight}

Being overweight increases the risk of heart disease and stroke and many other health problems, including high blood pressure, diabetes, gallstones and joint problems.

The increased risk of heart disease and stroke in overweight people is probably because they more commonly develop conditions which are all important risk factors in their own right - high blood pressure, high blood fat levels and diabetes.

Fat distribution is also important as a risk factor for cardiovascular disease and is usually classified as either android or gynoid. Android (apple shape) is characterised by the main deposits of fat being around the stomach, whereas gynoid (pear shape) is the main deposits of fat on the hips and thighs. People of 'apple shape' are at greater risk for the development of diabetes, hypertension (high blood pressure) or cardiovascular disease than people with a 'pear' shape.

Generally overweight is a combination of consuming more energy than the body needs and not using it up through being physically active.

\section{Diabetes}

Diabetes is characterised by raised blood sugar levels. It is a definite risk factor for cardiovascular disease. Diabetes is not a major risk factor for cardiovascular disease if it is mild and/or controlled, but is significant if severe or poorly 
controlled. Diabetes damages blood vessels generally and arteries tend to develop atherosclerosis as a result. The ultimate goal of the treatment of diabetes is to achieve optimum control of the disease.

\section{CONCLUSION}

There exists an intimate relationship between nutrition and cardiovascular disease, which is being well understood and studied in the developed world but is evolving rapidly in the developing world. The prevalence of this environmentally determined disease imposes a major burden on both societies. Many of the underlying causes and risk factors begin in youth. A heart -healthy lifestyle is the best defense against cardiovascular disease. The challenge is to promote healthy nutrition and physically active lifestyles as early as possible in children and young adults throughout the world. Many lifestyle habits begin during childhood. Thus, parents and families should encourage their children to make heart-healthy choices, such as following a healthy diet and being physically active. Make following a healthy lifestyle should be made the family goal.

\section{ACKNOWLEDGEMENT}

The corresponding author thanks the UGC for the grant of fellowship towards post doctoral studies

\section{REFERENCES}

1. Shanthi Mendis; Pekka Puska; Bo Norrving; World Health Organization (2011). Global Atlas on Cardiovascular Disease Prevention and Control (PDF). World Health Organization in collaboration with the World Heart Federation and the World Stroke Organization. pp. 3-18.
2. McNeill AM, Rosamond WD, Girman CJ, et al. The metabolic syndrome and 11-year risk of incident cardiovascular disease in the Atherosclerosis Risk in Communities Study. Diabetes Care. 2005; 28:385-390.

3. Kortelainen ML, Sarkioja T. Extent and composition of coronary lesions and degree of cardiac hypertrophy in relation to abdominal fatness in men under 40 years of age. Arterioscler Thromb Vasc Biol. 1997; 17:574-579.

4. Suwaidi JA, Wright RS, Grill JP, et al. Obesity is associated with premature occurrence of acute myocardial infarction. Clin Cardiol. 2001; 24:542-547.

5. O'Brien KD, Brehm BJ, Seeley RJ, et al. Diet-induced weight loss is associated with decreases in plasma serum amyloid A and C-reactive protein independent of dietary macronutrient composition in obese subjects. Journal of Clinical Endocrinology \& Metabolism. 2005; 90:2244-2249.

6. Kopp HP, Kopp CW, Festa A, et al. Impact of weight loss on inflammatory proteins and their association with the insulin resistance syndrome in morbidly obese patients. Arterioscler Thromb Vasc Biol. 2003; 23:1042-1047.

7. Wong ND, Thakral G, Franklin SS, et al. Preventing heart disease by controlling hypertension: impact of hypertensive subtype, stage, age, and sex. Am Heart J.2003; 145:888-895.

8. Liu S, Willett WC. Dietary glycemic load and atherothrombotic risk. Curr Atheroscler Rep. 2002; 4:454461.

9. Qiao Q, Jousilahti P, Eriksson J, Tuomilehto J. Predictive properties of impaired glucose tolerance for cardiovascular risk are not explained by the development of overt diabetes during follow-up. Diabetes Care. 2003; 26:2910-2914.

10. Gerich JE. The importance of tight glycemic control. Am J Med. 2005; 118:7S-11S.

11. Thompson PD, Lim V. Physical activity in the prevention of atherosclerotic coronary heart disease. Curr Treat Options Cardiovasc Med. 2003; 5:279-285.

12. Tanasescu M, Leitzmann MF, Rimm EB, Willett WC, Stampfer MJ, Hu FB. Exercise type and intensity in relation to coronary heart disease in men. JAMA. 2002; 288:1994-2000. 